\title{
A Note on the Appell Hypergeometric Matrix Function $\boldsymbol{F}_{\mathbf{2}}$
}

\author{
M. Hidan $\mathbb{D}^{1}$ and M. Abdalla $\mathbb{D}^{1,2}$ \\ ${ }^{1}$ Mathematics Department, Faculty of Science, King Khalid University, Abha, Saudi Arabia \\ ${ }^{2}$ Mathematics Department, Faculty of Science, South Valley University, Qena 83523, Egypt \\ Correspondence should be addressed to M. Hidan; mhedan@kku.edu.sa
}

Received 5 February 2020; Accepted 27 February 2020; Published 31 March 2020

Guest Editor: Shilpi Jain

Copyright (C) 2020 M. Hidan and M. Abdalla. This is an open access article distributed under the Creative Commons Attribution License, which permits unrestricted use, distribution, and reproduction in any medium, provided the original work is properly cited.

In this article, we introduce some of the mathematical properties of the second Appell hypergeometric matrix function $F_{2}\left(A, B_{1}\right.$, $\left.B_{2}, C_{1}, C_{2} ; z, w\right)$ including integral representations, transformation formulas, and series formulas.

\section{Introduction}

Appell defined and studied in [1-3] four kinds of double series of two variables $z, w$ as generalizations of the hypergeometric series:

$$
F(\alpha, \beta, \gamma ; z)=\sum_{n=0}^{\infty} \frac{(\alpha)_{n}(\beta)_{n}}{(\gamma)_{n}(1)_{n}} z^{n},
$$

where $z$ is a main variable in the unit disk $\{z \in \mathbb{C}:|z|<1\}, \alpha, \beta, \gamma$ are complex parameters with $\gamma \neq 0$, $-1,-2,-3, \ldots$, and $(\alpha)_{n}=\alpha(\alpha+1)(\alpha+2) \ldots(\alpha+n-1)$ $(n \in \mathbb{N})$ and $(\alpha)_{0}=1$. Here and throughout, let $\mathbb{C}$ and $\mathbb{N}$ denote the sets of complex numbers and positive integers, respectively, and let $\mathbb{N}_{0}=\mathbb{N} \cup\{0\}$.

Appell hypergeometric functions $F_{s}, s=\{1,2,3,4\}$ play an important role in mathematical physics in which broad practical applications can be found (see, e.g. [1, 3-7]). In particular, the Appell hypergeometric series $F_{2}$ arises frequently in various physical and chemical applications ([8-11]). The exact solutions of number of problems in quantum mechanics have been given $[6,7,9,12]$ in terms of Appell's function $F_{2}$. For readers, they can find some results of the classical second Appell hypergeometric function $F_{2}$ in [13-17].

On the other hand, many authors [18-25] generalized the hypergeometric series $F(\alpha, \beta, \gamma ; z)$ by extending parameters $\alpha, \beta$, and $\gamma$ to square matrices $A, B$, and $C$ in the complex space $\mathbb{C}^{d \times d}$. Recently, the extension of the classical Appell hypergeometric functions $F_{s}, s=\{1,2,3,4\}$, to the Appell hypergeometric matrix functions has been a subject of intensive studies [26-30]. The purpose of the present work is to study the second Appell hypergeometric matrix function $F_{2}\left(A, B_{1}, B_{2}, C_{1}, C_{2} ; z, w\right)$ on the domain $\left\{(z, w) \in \mathbb{C}^{2}:|z|+|w|<1\right\}$, with square matrix valued parameters $A, B_{1}, B_{2}, C_{1}$, and $C_{2}$ in $\mathbb{C}^{d \times d}$. We investigate some of the mathematical properties of this matrix function and introduce new integral representations, transformation formulas, and summation formulas.

\section{Some Known Definitions and Results}

We begin with a brief review of some definitions and notations. A matrix $E$ is a positive stable matrix in $\mathbb{C}^{d \times d}$ if $\operatorname{Re}(\lambda)$ $>0$ for all $\lambda \in \sigma(E)$, where $\sigma(E)$ is the set of all eigenvalues of $E$. $I$ and $\mathbf{0}$ stand for the identity matrix and the null matrix in $\mathbb{C}^{d \times d}$, respectively.

If $\Phi(z)$ and $\Psi(z)$ are holomorphic functions of the complex variable $z$, which are defined in an open set $\Omega$ of the complex plane and $E$ is a matrix in $\mathbb{C}^{d \times d}$ such that $\sigma(E) \subset \Omega$; then, from the properties of the matrix functional calculus [28], it follows that

$$
\Phi(E) \Psi(E)=\Psi(E) \Phi(E) .
$$

Hence, if $F$ in $\mathbb{C}^{d \times d}$ is a matrix for which $\sigma(F) \subset \Omega$ and also if $\mathrm{EF}=\mathrm{FE}$, then 


$$
\Phi(E) \Psi(F)=\Psi(F) \Phi(E) .
$$

By application of the matrix functional calculus, for $E$ in $\mathbb{C}^{d \times d}$, then from $[23,31]$, the Pochhammer symbol or shifted factorial defined by

$$
(E)_{n}= \begin{cases}E(E+I) \ldots(E+(n-1) I)=\Gamma^{-1}(E) \Gamma(E+n I), & n \in \mathbb{N}, \\ I, & n=0,\end{cases}
$$

with the condition

$$
E+n I \text { is invertible for all integers } n \in \mathbb{N}_{0} .
$$

From (5), it is easy to find that

$$
\begin{aligned}
& (E)_{n-k}=(-1)^{k}(E)_{n}\left[(I-E-n I)_{k}\right]^{-1} ; \quad 0 \leq k \leq n, \\
& (E)_{m+n}=(E)_{n}(E+n I)_{m} \text { or }(E)_{m+n}=(E)_{m}(E+m I)_{n} .
\end{aligned}
$$

From [28], one obtains

$$
\frac{(-1)^{k}}{(n-k) !} I=\frac{(-n)_{k}}{n !} I=\frac{(-n I)_{k}}{n !} ; \quad 0 \leq k \leq n .
$$

Definition 1 (see [31]). If $E$ is a matrix in $\mathbb{C}^{d \times d}$, such that $\operatorname{Re}(z)>0$ for all eigenvalues $z$ of $E$, then $\Gamma(E)$ is well defined as

$$
\begin{aligned}
\Gamma(E) & =\int_{0}^{\infty} \tau^{E-I} e^{-\tau} \mathrm{d} \tau, \\
\tau^{E-I} & =\exp ((E-I) \ln \tau) .
\end{aligned}
$$

Definition 2 (see [31]). If $E$ and $F$ are positive stable matrices in $\mathbb{C}^{d \times d}$ and $\mathrm{EF}=\mathrm{FE}$, then the Beta matrix function is well defined by

$$
\mathscr{B}(E, F)=\int_{0}^{1} \tau^{E-I}(1-\tau)^{F-I} \mathrm{~d} \tau=\Gamma^{-1}(E+F) \Gamma(E) \Gamma(F) .
$$

Definition 3 (see[23]). Suppose that $N_{1}, N_{2}$, and $N_{3}$ are matrices in $\mathbb{C}^{d \times d}$, such that $N_{3}$ satisfies condition (5). Then, the hypergeometric matrix function ${ }_{2} F_{1}\left(N_{1}, N_{2} ; N_{3} ; z\right)$ is given by

$$
{ }_{2} F_{1}\left(N_{1}, N_{2} ; N_{3} ; z\right)=\sum_{n \geq 0} \frac{\left(N_{1}\right)_{n}\left(N_{2}\right)_{n}\left[\left(N_{3}\right)_{n}\right]^{-1}}{n !} z^{n}
$$

Definition 4. If $E$ is the positive stable matrix in $\mathbb{C}^{d \times d}$, then the Laguerre-type matrix polynomial is defined by [28]

$$
\begin{aligned}
L_{n}^{E}(z) & =\sum_{k=0}^{n} \frac{(-1)^{k}(z)^{k}}{k !(n-k) !}(E+I)_{n}(E+I)_{k}^{-1} \\
& =\frac{(E+I)_{n}}{n !}{ }_{1} F_{1}(-n I ; E+I ; z), \quad n \in \mathbb{N}_{0},
\end{aligned}
$$

where ${ }_{1} F_{1}$ is the confluent hypergeometric matrix function (cf. [25]).

Definition 5 (see[28, 32, 33]). Let $E$ and $F$ be positive stable matrices in $\mathbb{C}^{d \times d}$, then the Jacobi matrix polynomial $\mathbb{P}_{n}^{(E, F)}(z)$ is defined by

$$
\begin{aligned}
\mathbb{P}_{n}^{(E, F)}(z)= & \sum_{k=0}^{n}\left(\begin{array}{c}
n \\
k
\end{array}\right) \Gamma^{-1}(E+F+(n+1) I) \Gamma^{-1}(F+(k+1) I) \\
& \Gamma(F+(n+1) I) \Gamma(E+F+(n+k+1) I) \frac{(-1)^{n+k}(1+z)^{k}}{2^{k} n !}, \\
= & \frac{(E+I)_{n}}{n !}{ }_{2} F_{1}\left(-n I, E+F+(n+1) I ; E+I ; \frac{1-z}{2}\right) .
\end{aligned}
$$

Using (6) and (11), we can write the second kind of two complex variables Appell hypergeometric matrix function in the following definition (see $[26,28]$ ).

Definition 6. Let $A, B_{1}, B_{2}, C_{1}$, and $C_{2}$ be commutative matrices in $\mathbb{C}^{d \times d}$ with $C_{1}+k I$ and $C_{2}+k I$ being invertible for all integers $k \in \mathbb{N}_{0}$. Then, the second Appell hypergeometric matrix function $F_{2}\left(A, B_{1}, B_{2}, C_{1}, C_{2} ; z, w\right)$ is defined in the following form:

$$
\begin{aligned}
F_{2}\left(A, B_{1}, B_{2}, C_{1}, C_{2} ; z, w\right) & =\sum_{s_{1}, s_{2}=0}^{\infty}(A)_{s_{1}+s_{2}}\left(B_{1}\right)_{s_{1}}\left(B_{2}\right)_{s_{2}}\left[\left(C_{1}\right)_{s_{1}}\right]^{-1}\left[\left(C_{2}\right)_{s_{2}}\right]^{-1} \frac{z^{s_{1}} w^{s_{2}}}{s_{1} ! s_{2} !} \\
& =\sum_{s_{1}=0}^{\infty}(A)_{s_{1}}\left(B_{1}\right)_{s_{1}}\left[\left(C_{1}\right)_{s_{1}}\right]_{2}^{-1}{ }_{2} F_{1}\left(A+s_{1} I, B_{2} ; C_{2} ; w\right) \frac{z^{s_{1}}}{s_{1} !}, \quad(|z|+|w|<1) .
\end{aligned}
$$

\section{Main Results}

In this section, we investigate some of the main properties of the second Appell hypergeometric matrix function $F_{2}\left(A, B_{1}\right.$, $\left.B_{2}, C_{1}, C_{2} ; z, w\right)$ such as integral representations, transformation formulas, and summation formulas

\subsection{Integral Representations}

Theorem 1. Let $A, C_{1}$, and $C_{2}$ be positive stable matrices in $\mathbb{C}^{d \times d}$. Then, for $|z|+|w|<1$, then the function $F_{2}\left(A, B_{1}, B_{2}, C_{1}\right.$, $\left.C_{2} ; z, w\right)$ defined in (14) can be represented in the following integer forms: 


$$
\begin{aligned}
& F_{2}\left(A, B_{1}, B_{2}, C_{1}, C_{2} ; z, w\right)=\Gamma^{-1}(A) \\
& \quad \times \int_{0}^{\infty} u^{A-I} e^{-u}{ }_{1} F_{1}\left(B_{1} ; C_{1} ; z u\right)_{1} F_{1}\left(B_{2} ; C_{2} ; w u\right) \mathrm{d} u \\
& F_{2}\left(A,-m I,-n I, C_{1}+I, C_{2}+I ; z, w\right) \\
& =m ! n !\left[\left(C_{1}+I\right)_{m}\right]^{-1}\left[\left(C_{2}+I\right)_{n}\right]^{-1} \Gamma^{-1}(A) \\
& \quad \times \int_{0}^{\infty} u^{A-I} e^{-u} L_{m}^{C_{1}}(z u) L_{n}^{C_{2}}(w u) \mathrm{d} u
\end{aligned}
$$

Proof. Replacing the Pochhammer symbol $(A)_{m+n}$ in definition (14) by its integral representation which is obtained from (5) and (9), we get the desired result (15).

Using integral formula (15) and the relation given in (12), we have

$$
\begin{aligned}
F_{2}\left(A,-m I,-n I, C_{1}+I, C_{2}+I ; z, w\right) & =\Gamma^{-1}(A) \int_{0}^{\infty} u^{A-I} e^{-u}{ }_{1} F_{1}\left(-m I ; C_{1}+I ; z u\right)_{1} F_{1}\left(-n I ; C_{2}+I ; w u\right) \mathrm{d} u \\
& =m ! n !\left[\left(C_{1}+I\right)_{m}\right]^{-1}\left[\left(C_{2}+I\right)_{n}\right]^{-1} \Gamma^{-1}(A) \times \int_{0}^{\infty} u^{A-I} e^{-u} L_{m}^{C_{1}}(z u) L_{n}^{C_{2}}(w u) \mathrm{d} u
\end{aligned}
$$

which completes proof relation (16).

Theorem 2. For the matrix function $F_{2}\left(A, B_{1}, B_{2}, C_{1}, C_{2} ; z\right.$, $w)$, we have the following transformations:

\subsection{Transformation Formulas}

$$
\begin{aligned}
& F_{2}\left(A, B_{1}, B_{2}, C_{1}, C_{2} ; z, w\right)=(1-z)^{-A} F_{2}\left(A, C_{1}-B_{1}, B_{2}, C_{1}, C_{2} ; \frac{-z}{1-z}, \frac{w}{1-w}\right) \\
& F_{2}\left(A, B_{1}, B_{2}, C_{1}, C_{2} ; z, w\right)=(1-w)^{-A} F_{2}\left(A, B_{1}, C_{2}-B_{2}, C_{1}, C_{2} ; \frac{z}{1-w}, \frac{w}{w-1}\right) \\
& F_{2}\left(A, B_{1}, B_{2}, C_{1}, C_{2} ; z, w\right)=(1-z-w)^{-A} F_{2}\left(A, C_{1}-B_{1}, C_{2}-B_{2}, C_{1}, C_{2} ; \frac{-z}{1-z-w}, \frac{-w}{1-z-w}\right),
\end{aligned}
$$

where $A, B_{1}, B_{2}, C_{1}$, and $C_{2}$ are commutative matrices in $\mathbb{C}^{d \times d}$ with $C_{1}+k I$ and $C_{2}+k I$ being invertible for all integer $k \in \mathbb{N}_{0}$, and $B_{1}, B_{2}, C_{1}, C_{2}, C_{1}-B 1$, and $C_{2}-B_{2}$ are positively stable.

Proof. We will prove only (18) since the others can be proved similarly. Using matrix Kummer's first formula (cf. [8]),

$$
{ }_{1} F_{1}(B ; C ; z)=e^{z}{ }_{1} F_{1}(C-B ; C ;-z),
$$

in (15), we have

$$
\begin{aligned}
& F_{2}\left(A, B_{1}, B_{2}, C_{1}, C_{2} ; z, w\right)=\Gamma^{-1}(A) \\
& \quad \times \int_{0}^{\infty} u^{A-I} e^{-(1-z) u}{ }_{1} F_{1}\left(C_{1}-B_{1} ; C_{1} ; z u\right){ }_{1} F_{1}\left(B_{2} ; C_{2} ; w u\right) \mathrm{d} u
\end{aligned}
$$
(18).

Substituting $t=(1-z) u$ into (22), we obtain formula

Now, connections with the Gauss hypergeometric matrix function is considered by the following theorem:
Theorem 3. Let $F_{2}\left(A, B, B^{\prime}, C_{1}, C_{2} ; z, w\right)$ be given in (14). The following formulas hold true:

$F_{2}\left(A, B_{1}, B_{2}, C_{1}, C_{2} ; 0, w\right)={ }_{2} F_{1}\left(A, B_{2} ; C_{2} ; w\right)$,

$F_{2}\left(A, B_{1}, B_{2}, C_{1}, C_{2} ; z, 0\right)={ }_{2} F_{1}\left(A, B_{1} ; C_{1} ; z\right)$,

$F_{2}\left(A, \mathbf{0}, B_{2}, C_{1}, C_{2} ; z, w\right)={ }_{2} F_{1}\left(A, B_{2} ; C_{2} ; z\right)$

$F_{2}\left(A, B_{1}, \mathbf{0}, C_{1}, C_{2} ; z, w\right)={ }_{2} F_{1}\left(A, B_{1} ; C_{1} ; z\right)$

$F_{2}\left(A, \mathbf{0}, B_{2}, C_{1}, C_{2} ; z, 0\right)=(1-z)^{-A}{ }_{2} F_{1}\left(A, B_{2} ; C_{2} ; \frac{z}{1-z}\right)$,

$F_{2}\left(A, B_{1}, \mathbf{0}, C_{1}, C_{2} ; 0, w\right)=(1-w)^{-A}{ }_{2} F_{1}\left(A, B_{1} ; C_{1} ; \frac{w}{1-w}\right)$

where ${ }_{2} F_{1}$ is the Gauss hypergeometric matrix function defined in (11). 
Proof. The proof of (23)-(26) is a direct consequence of definition (27). The relation (27) is obtained setting $C_{1}=B_{1}$ in (18) and then using (25). Similarly, the relation (28) is derived setting $C_{2}=B_{2}$ in (19) and then using (26).

3.3. Some Summation Formulas. We now present the summation formulas behavior of the second Appell hypergeometric matrix function $F_{2}\left(A, B_{1}, B_{2}, C_{1}, C_{2} ; z, w\right)$ by the following results.

Theorem 4. The following finite summation formula holds true:

$$
\begin{aligned}
\sum_{n=0}^{\mu} \frac{(\mathbf{C}+I)_{n}}{n !} F_{2}(\mathbf{A},-n I,-n I, \mathbf{C}+I, \mathbf{C}+I ; z, w) \\
=\frac{(\mathbf{A}-I)^{-1}(\mathbf{C}+I)_{\mu+1}}{(z-w) \mu !}\left\{F_{2}(\mathbf{A}-I,-\mu I,-(\mu+1) I, \mathbf{C}\right. \\
\quad+I, \mathbf{C}+I ; z, w)+z \rightleftharpoons w\},
\end{aligned}
$$

where $\boldsymbol{A}$ and $\boldsymbol{C}$ are positively stable in $\mathbb{C}^{d \times d}$ and $z \rightleftharpoons w$ indicates the presence of a second term that originates from the first by interchanging $z$ and $w$.

Proof. Using (16), we find that

$$
\begin{aligned}
& \sum_{n=0}^{\mu} \frac{(\mathbf{C}+I)_{n}}{n !} F_{2}(\mathbf{A},-n I,-n I, \mathbf{C}+I, \mathbf{C}+I ; z, w) \\
& \quad=\Gamma^{-1}(\mathbf{A}) \sum_{n=0}^{\mu} n !\left[(\mathbf{C}+I)_{n}\right]^{-1} \int_{0}^{\infty} u^{\mathbf{A}-I} e^{-u} L_{\mu}^{\mathrm{C}}(z u) L_{n}^{\mathrm{C}}(w u) \mathrm{d} u .
\end{aligned}
$$

By interchanging the order of summation and integration and applying the following formula [28]:

$$
\begin{aligned}
\sum_{n=0}^{\mu} n ! & {\left[(\mathbf{C}+I)_{n}\right]^{-1} L_{n}^{\mathrm{C}}(z) L_{n}^{\mathrm{C}}(w) } \\
= & (\mu+1) !\left[(\mathbf{C}+I)_{\mu}\right]^{-1}(z-w)^{-1}\left\{L_{\mu}^{\mathrm{C}}(z) L_{\mu+1}^{\mathrm{C}}(w)\right. \\
& \left.-L_{\mu+1}^{\mathrm{C}}(z) L_{\mu}^{\mathrm{C}}(w)\right\},
\end{aligned}
$$

and then taking into consideration (16), we obtain formula (29).

To extend this theorem, we propose to obtain some more formulas centering around the Appell's matrix function $F_{2}$; it follows that

Theorem 5. Suppose that $\boldsymbol{A}$ and $\boldsymbol{B}$ are positively stable in $\mathbb{C}^{d \times d}$ such that $\boldsymbol{B}$ satisfies spectral condition (5), with $|t|<1,|z t /((1-w)(1-t))|<1$ and $|w /((1-w)(1-t))|<1$. The following generating matrix function holds true:

$$
\begin{aligned}
\sum_{n=0}^{\infty} \frac{(\mathbf{B}+I)_{\mu+n}}{n !} F_{2}(\mathbf{A},-n I,-(n+\mu) I ; \mathbf{B}+I, \mathbf{B}+I ; z, w) t^{n} \\
=(\mathbf{B}+I)_{\mu}(1-w)^{-\mathbf{A}}(1-t)^{-(\mathbf{B}+(1+\mu) I)} \\
\quad \times F_{4}\left(\mathbf{A}, \mathbf{B}+(1+\mu) I ; \mathbf{B}+I, \mathbf{B}+I ; \frac{-z t}{(1-w)(1-t)}\right. \\
\left.\quad \frac{-w}{(1-w)(1-t)}\right)
\end{aligned}
$$

where $F_{4}$ is the four Appell's matrix function defined in [27-29].

Proof. To prove (32), we require formula (19) and the relations (12); thus, we have

$$
\begin{aligned}
\sum_{n=0}^{\infty} & \frac{(\mathbf{B}+I)_{\mu+n}}{n !} F_{2}(\mathbf{A},-n I,-(n+\mu) I ; \mathbf{B}+I, \mathbf{B}+I ; z, w) t^{n} \\
= & (1-w)^{-\mathbf{A}} \times \sum_{n=0}^{\infty} \frac{(\mathbf{B}+I)_{\mu+n}}{n !} F_{2}(\mathbf{A},-n I ; \mathbf{B}+(\mu+n+1) I ; \\
& \left.\mathbf{B}+I, \mathbf{B}+I ; \frac{z}{(1-w)} ; \frac{w}{(w-1)}\right) \\
= & (1-w)^{-\mathbf{A}} \sum_{s, n=0}^{\infty} \sum_{r=0}^{n}(\mathbf{A})_{s+r}\left[(\mathbf{B}+I)_{r}\right]^{-1}\left[(\mathbf{B}+I)_{s}\right]^{-1} \\
& \cdot(\mathbf{B}+I)_{\mu+n+r} \times \frac{t^{n}}{(n-r) ! s ! r !}\left(\frac{-z}{(1-w)}\right)^{r}\left(\frac{-w}{(1-w)}\right)^{s} \\
= & (1-w)^{-\mathbf{A}} \sum_{s, r=0}^{\infty} \frac{1}{s ! r !}(\mathbf{A})_{s+r}\left[(\mathbf{B}+I)_{r}\right]^{-1}\left[(\mathbf{B}+I)_{s}\right]^{-1} \\
& \cdot(\mathbf{B}+I)_{\mu+s+r} \\
& \left.\cdot \frac{-w}{(1-w)(1-t)}\right) \cdot \\
& \times\left(\frac{-z t}{(1-w)}\right)^{r}\left(\frac{-w}{(1-w)}\right)^{s} \sum_{n=0}^{\infty} \frac{(\mathbf{B}+(1+\mu+s+r) I)_{n}}{n !} \\
= & (\mathbf{B}+I)_{\mu}(1-w)^{-\mathbf{A}}(1-t)^{-(\mathbf{B}+(1+\mu) I)} \\
\times F_{4}\left(\mathbf{A}, \mathbf{B}+(1+\mu) I ; \mathbf{B}+I, \mathbf{B}+I ; \frac{-z t}{(1-w)(1-t)}\right. & \\
& (1-w) \\
&
\end{aligned}
$$

This completes the proof of Theorem 5 . 
Putting $\mu=0$ and then using the following formula,

$$
F_{4}(\mathbf{A}, \mathbf{B} ; \mathbf{B}, \mathbf{B} ; z, w)=(1-z-w)^{-\mathbf{A}}{ }_{2} F_{1}\left(\frac{\mathbf{A}}{2}, \frac{1}{2}(\mathbf{A}+I) ; \mathbf{B} ; \frac{4 z w}{(1-z-w)^{2}}\right)
$$

Thus, (32) reduces to

$$
\begin{aligned}
\sum_{n=0}^{\infty} & \frac{(\mathbf{B}+I)_{\mu+n}}{n !} F_{2}(\mathbf{A},-n I,-(n+\mu) I ; \mathbf{B}+I, \mathbf{B}+I ; z, w) t^{n}=(1-w)^{-\mathbf{A}} \\
& \times \sum_{n=0}^{\infty} \frac{(\mathbf{B}+I)_{\mu+n}}{n !} F_{2}\left(\mathbf{A},-n I ; \mathbf{B}+(\mu+n+1) I ; \mathbf{B}+I, \mathbf{B}+I ; \frac{z}{(1-w)} ; \frac{w}{(w-1)}\right) \\
= & (1-t)^{\mathbf{A}-(\mathbf{B}+I)}[1-(1-z-w) t]^{-\mathbf{A}}{ }_{2} F_{1}\left(\frac{\mathbf{A}}{2}, \frac{1}{2}(\mathbf{A}+I) ; \mathbf{B}+I ; \frac{4 z w t}{[1-(1-z-w) t]^{2}}\right) .
\end{aligned}
$$

Now, we shall see that (32) and (35) enable us to obtain some new formulas. By (29), we have

$$
\begin{aligned}
\sum_{n=0}^{\infty} \mathbf{A}^{-1} & \frac{(\mathbf{B}+I)_{n+1}}{(z-w) n !}\left[F_{2}(\mathbf{A}-I,-n I,-(n+1) I ; \mathbf{B}+I, \mathbf{B}+I ; z, w)\right. \\
& +z \rightleftharpoons w] t^{n} \\
= & \sum_{n=0}^{\infty} \sum_{r=0}^{n} \frac{(\mathbf{B}+I)_{k}}{k !} F_{2}(\mathbf{A},-k I ;-k I ; \mathbf{B}+I, \mathbf{B}+I ; z, w) t^{n}
\end{aligned}
$$

Using (35), we arrive at

$$
\begin{aligned}
\sum_{n=0}^{\infty} \frac{(\mathbf{B}+I)_{n+1}}{n !}\left[F_{2}(\mathbf{A}-I,-n I,-(n+1) I ; \mathbf{B}+I, \mathbf{B}+I ; z, w)\right. \\
\quad+z \rightleftharpoons w] t^{n} \\
=F_{2}(\mathbf{A},-k I ;-k I ; \mathbf{B}+I, \mathbf{B}+I ; z, w) t^{n} \\
=\mathbf{A}(z-w)(1-t)^{\mathbf{A}-(\mathbf{B}+2 I)}[1-(1-z-w) t]^{-\mathbf{A}} \\
\quad \times{ }_{2} F_{1}\left(\frac{\mathbf{A}}{2}, \frac{1}{2}(\mathbf{A}+I) ; \mathbf{B}+I ; \frac{4 z w t}{[1-(1-z-w) t]^{2}}\right) .
\end{aligned}
$$

With the help of a generating function for Jacobi matrix polynomials (see $[28,32,33]$ ),

$$
\begin{aligned}
\sum_{n=0}^{\infty}(\mathbf{B}+\mathbf{C}+I)_{n}\left[(\mathbf{B}+I)_{n}\right]^{-1} \mathbb{P}_{n}^{(\mathbf{B}, \mathbf{C})}(z) t^{n} \\
=(1-t)^{-(\mathbf{B}+\mathbf{C}+I)}{ }_{2} F_{1}\left(\frac{1}{2}(\mathbf{B}+\mathbf{C}+I), \frac{1}{2}(\mathbf{B}+\mathbf{C}+2 I) ; \mathbf{B}\right. \\
\left.\quad+I ; \frac{2 t(z-1)}{(1-t)^{2}}\right) .
\end{aligned}
$$

We rewrite (35) as

$$
\begin{aligned}
& \sum_{n=0}^{\infty} \frac{(\mathbf{B}+I)_{n+1}}{n !}\left[F_{2}(\mathbf{B}+\mathbf{C},-n I,-(n+1) I ; \mathbf{B}+I, \mathbf{B}+I ; z, w)\right. \\
& \quad+z \rightleftharpoons w] t^{n} \\
& =(\mathbf{B}+\mathbf{C}+I)(z-w)(1-t)^{\mathbf{C}-I} \sum_{r=0}^{\infty}(\mathbf{B}+\mathbf{C}+I)_{r}\left[(\mathbf{B}+I)_{r}\right]^{-1} \\
& \quad \times \mathbb{P}_{r}^{(\mathbf{B}, \mathbf{C})}\left(\frac{(1-w)(1-z)+z w}{(1-z-w)}\right)(1-z-w)^{r} t^{r},
\end{aligned}
$$

which yields

$$
\begin{aligned}
& \mathbb{P}_{n}^{(\mathbf{B}, \mathbf{C})}\left(\frac{(1-w)(1-z)+z w}{(1-z-w)}\right)=(\mathbf{B}+I)_{n}(\mathbf{B}+\mathbf{C}+I) \\
& \cdot\left[(\mathbf{B}+\mathbf{C}+I)_{n}\right]^{-1} \\
& \quad \times(z-w)^{-1}(1-z-w)^{-n} \sum_{r=0}^{n} \frac{(\mathbf{C}-I)_{r}}{(n-r) !} \frac{(\mathbf{B}+I)_{r+1}}{r !} \\
& \quad \times\left[F_{2}(\mathbf{B}+\mathbf{C},-r I,-(r+1) I ; \mathbf{B}+I, \mathbf{B}+I ; z, w)+z \rightleftharpoons w\right] .
\end{aligned}
$$

\section{Data Availability}

No data were used to support this study.

\section{Conflicts of Interest}

The authors declare that they have no conflicts of interest. 


\section{Authors' Contributions}

All the authors contributed equally and significantly to writing of this article. All the authors read and approved the final manuscript.

\section{Acknowledgments}

The authors wish to acknowledge the approval and the support of this research study from the Deanship of Scientific Research in King Khalid University, Abha, Saudi Arabia under grant (R.G.P-79-41).

\section{References}

[1] P. Appell, "Sur les fonctions hypergéométriques de deux variables," Journal de Mathématiques Pures et Appliquées, vol. 8, pp. 173-216, 1882.

[2] P. Appell, "Sur les fonctions hypergéometriques de plusieurs variables," The Mathematical Heritage of Henri Poincare, vol. 10, 1925.

[3] P. Appell and J. Kampé de Fériet, "Fonctions hypergé ométrique's et hypersphériques; Polynômes d'Hermite," 1926.

[4] H. Exton, Multiple Hypergeometric Functions and Applications, Wiley, New York, NY, USA, 1976.

[5] A. M. Mathai and H. Haubold, Special Functions for Applied Scientists, Springer Science, New York, NY, USA, 2008.

[6] T. SadyKov, Hypergeomatric Functions in Several Complex Variables, Ph.D. thesis, Stockholm University, Stockholm, Sweden, 2002.

[7] L. J. Slater, Generalized Hypergeometric Functions, Cambridge University Press, Cambridge, UK, 1966.

[8] J. Fleischer, F. Jegerlehner, and O. V. Tarasov, "A new hypergeometric representation of one-loop scalar integrals in d dimensions," Nuclear Physics B, vol. 672, no. 1-2, pp. 303-328, 2003.

[9] L. D. Landau and E. M. Lifshitz, Quantum Mechanics: Nonrelativistic Theory, Pergamon Press, London, UK, 1981.

[10] N. Saad and R. L. Hall, "Integrals containing confluent hypergeometric functions with applications to perturbed singular potentials," Journal of Physics A: Mathematical and General, vol. 36, no. 28, pp. 7771-7788, 2003.

[11] N. Saad and R. L. Hall, "Closed-form sums for some perturbation series involving hypergeometric functions," Journal of Physics A: Mathematical and General, vol. 35, no. 18, pp. 4105-4123, 2002.

[12] V. F. Tarasov, "The generalization of slater's and marvin's integrals and their representations by means of appell's functions $f_{2}(x, y)$," Modern Physics Letters B, vol. 8, no. 23, pp. 1417-1426, 1994.

[13] Yu. A. Brychkov and N. Saad, "Some formulas for the Appell function $F_{2}\left(a, b, b^{\prime} ; c ; w, z\right)$," Integral Transforms and Special Functions, vol. 23, pp. 793-802, 2014.

[14] A. Erdélyi, W. Magnus, F. Oberhettinger, and F. Tricomi, Higher Transcendental Functions. Vol. I. Based on Notes Left by Harry Bateman. With a Preface by Mina Rees. With a Foreword by E.C. Watson. Reprint of the 1953 Original, Robert E. Krieger Publishing Co. Inc., Melbourne, FL, USA, 1981.

[15] X. Wang, "Recursion formulas for Appell functions," Integral Transforms and Special Functions, vol. 23, no. 6, pp. 421-433, 2012.
[16] X. Wang, "Infinite summation formulas of double hypergeometric functions," Integral Transforms and Special Functions, vol. 27, no. 5, pp. 347-364, 2016.

[17] X. Wang and Y. Chen, "Finite summation formulas of double hypergeometric functions," Integral Transforms and Special Functions, vol. 28, no. 3, pp. 239-253, 2017.

[18] M. Abdalla, "Further results on the generalised hypergeometric matrix functions," International Journal of Computing Science and Mathematics, vol. 10, no. 1, pp. 1-10, 2019.

[19] M. Abdalla and A. Bakhet, "Extended Gauss hypergeometric matrix functions," Iranian Journal of Science and Technology, Transactions A: Science, vol. 42, no. 3, pp. 1465-1470, 2018.

[20] M. Abdalla, "On the incomplete hypergeometric matrix functions," The Ramanujan Journal, vol. 43, no. 3, pp. 663678, 2017.

[21] A. Bakhet, Y. Jiao, and F. He, "On the Wright hypergeometric matrix functions and their fractional calculus," Integral Transforms and Special Functions, vol. 30, no. 2, pp. 138-156, 2019.

[22] He. Fuli, A. Bakhet, M. Hidan, and M. Abdalla, "On the extended hypergeometric matrix functions and their applications for the derivatives of the extended jacobi matrix polynomial," Mathematical Problems in Engineering, vol. 25, 2020, in Press.

[23] L. Jódar and J. C. Cortés, "On the hypergeometric matrix function," Journal of Computational and Applied Mathematics, vol. 99, no. 1-2, pp. 205-217, 1998.

[24] S. Z. Rida, M. Abul-Dahab, M. A. Saleem, and M. T. Mohammed, "On Humbert matrix function $\Psi_{1}(A, B ; C$, $\left.C^{\prime} ; z, w\right)$ of two complex variables under differential operator," International Journal of Industrial Mathematics, vol. 32, pp. 167-179, 2010.

[25] G. Wehowar and E. Hausenblas, "The second Kummer function with matrix parameters and its asymptotic behaviour," Abstract and Applied Analysis, vol. 2018, Article ID 7534651, p. 8, 2018.

[26] A. Altin, B. Çekim, and R. Şahin, "On the matrix versions of Appell hypergeometric functions," Quaestiones Mathematicae, vol. 37, no. 1, pp. 31-38, 2014.

[27] H. Abd-Elmageed, M. Abdalla, M. Abul-Ez, and N. Saad, "Some results on the first Appell matrix function $F_{1}(A, B, B$, $C ; z, w)$," Linear and Multilinear Algebra, vol. 68, no. 2, pp. 278-292, 2020.

[28] M. Abdalla, "Special matrix functions: characteristics, achievements and future directions," Linear and Multilinear Algebra, vol. 68, no. 1, pp. 1-28, 2020.

[29] R. Dwivedi and V. Sahai, "A note on the Appell matrix functions," Quaestiones Mathematicae, vol. 29, 2019.

[30] A. Verma and S. Yadav, On the Incomplete Second Appell Hypergeometric Matrix Functions Linear and Multilinear Algebra, vol. 69, 2020.

[31] L. Jódar and J. C. Cortés, "Some properties of Gamma and Beta matrix functions," Applied Mathematics Letters, vol. 11, no. 1, pp. 89-93, 1998.

[32] B. Çekim, A. Altin, and R. Aktaş, "Some new results for Jacobi matrix polynomials,” Filomat, vol. 27, no. 4, pp. 713-719, 2013.

[33] F. Taşdelen, B. Çekim, and R. Aktaş, "On a multivariable extension of Jacobi matrix polynomials," Computers \& Mathematics with Applications, vol. 61, pp. 2412-2423, 2011. 